\title{
Validation of the Korean Version of the Bronchiectasis Health Questionnaire
}

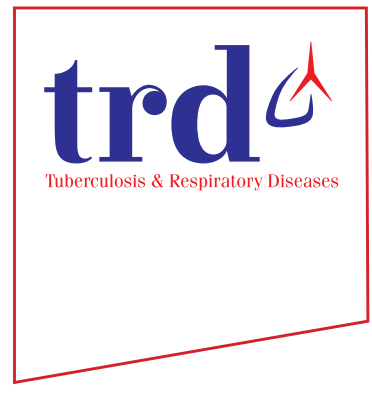

\author{
Hyun Kuk Kim, M.D. ${ }^{1}{ }^{(i)}$, Hyun Lee, M.D. ${ }^{2}$, Sang-Heon Kim, M.D. ${ }^{2}$, Hayoung Choi, M.D. ${ }^{3}$, Jae Ha
} Lee, M.D. ${ }^{1}$, Jae Seung Lee, M.D. ${ }^{4}$, Sei Won Lee, M.D. ${ }^{4}$ and Yeon-Mok Oh, M.D. ${ }^{4}$ (D)

${ }^{1}$ Department of Pulmonary and Critical Care Medicine, Inje University Haeundae Paik Hospital, Inje University College of Medicine, Busan, ${ }^{2}$ Division of Pulmonary Medicine and Allergy, Department of Internal Medicine, Hanyang University College of Medicine, Seoul, ${ }^{3}$ Division of Pulmonary, Allergy, and Critical Care Medicine, Department of Internal Medicine, Hallym University Kangnam Sacred Heart Hospital, Seoul, ${ }^{4}$ Department of Pulmonary and Critical Care Medicine, Asan Medical Center, University of Ulsan College of Medicine, Seoul, Korea

Background: The Bronchiectasis Health Questionnaire (BHQ) is a simple and repeatable, self-reporting health status questionnaire for bronchiectasis. We have translated the original version of the BHQ into Korean using a standardized methodology. The purpose of this study was to assess the validity of the Korean version of the BHQ (K-BHQ) with Korean patients.

Methods: Stable state patients with bronchiectasis from two academic hospitals were enrolled in this study. The validity was assessed by investigating the relationship between the K-BHQ scores and the Korean version of the Chronic Obstructive Pulmonary Disease Assessment Test (K-CAT) scores. We also investigated the relationship between the K-BHQ scores and other variables of the modified Medical Research Council's (mMRC) dyspnea scale, lung function, and exacerbations.

Results: A total of 126 patients with bronchiectasis were enrolled. The mean age was 64.3 (standard deviation [SD], 9.7). Women comprised $53.2 \%$ of the patients. The mean forced expiratory volume in one second $\left(\mathrm{FEV}_{1}\right)$ was $60 \%$ of the predicted value (SD, 18.9\%); the mean K-CAT score was 17.6 (SD, 9.1). The K-BHQ scores correlated strongly with the K-CAT scores $(\mathrm{r}=-0.656, \mathrm{p}<0.001)$. There was significant correlation between the K-BHQ scores and the mMRC dyspnea scale $(\rho=-0.409, \mathrm{p}<0.001), \mathrm{FEV}_{1}(\mathrm{r}=0.406, \mathrm{p}<0.001)$, and number of exacerbations requiring hospitalization $(\rho=-0.303$, $\mathrm{p}=0.001)$.

Conclusion: The K-BHQ is valid for assessing the health-related quality of life or health status of Korean bronchiectasis patients.

Keywords: Bronchiectasis; Bronchiectasis Health Questionnaire; Validation

Address for correspondence: Yeon-Mok Oh, M.D.

Department of Pulmonary and Critical Care Medicine, Asan Medical Center, University of Ulsan College of Medicine, 88 Olympic-ro 43-gil, Songpa-gu, Seoul 05505, Korea

Phone: 82-2-3010-3136, Fax: 82-2-3010-6968, E-mail: ymoh55@amc.seoul.kr

Received: Mar. 16, 2020, Revised: Mar. 20, 2020, Accepted: Mar. 30, 2020, Published online: Apr. 29, 2020

@(t) is identical to the Creative Commons Attribution Non-Commercial License (http://creativecommons.org/licenses/by-nc/4.0/). 


\section{Introduction}

Bronchiectasis is a chronic airway disease characterized by a clinical syndrome that includes cough, sputum production, hemoptysis, and bronchial infection. Radiographic images illustrate permanent dilatation and a thickened wall of the bronchi ${ }^{1}$. Bronchiectasis is increasing in prevalence and escalates the public health burden, resulting in increased health care costs, hospitalization rates, and mortality ${ }^{2,3}$. A recent study in Korea using a government health insurance database showed that the prevalence of bronchiectasis in Korea was higher than that in other Western countries, and the healthcare cost was high ${ }^{4}$. In addition to the high prevalence and economic burden, assessment of health-related quality of life (HRQOL) is important in chronic diseases such as bronchiectasis, as it can evaluate the overall impact on health from the patient's point of view. HRQOL is severely impaired in bronchiectasis ${ }^{5}$. The health status of bronchiectasis patients can be evaluated with validated questionnaires, such as the St. George's Respiratory Questionnaire (SGRQ), Leicester Cough Questionnaire, and Quality of Life-Bronchiectasis (QoL-B) $)^{6-8}$. However, these questionnaires are not practical for general use in the management of bronchiectasis patients as they are too lengthy, and the scoring system is very complicated. Spinou et al. ${ }^{9}$ recently developed a shorter and more practical tool, termed the Bronchiectasis Health Questionnaire (BHQ). Despite the questionnaire's simplicity, the authors concluded that the questionnaire works well in a different population from the development cohort. The authors also presented a translation of the questionnaire into 11 languages with linguistic validation ${ }^{9}$. Given the significance of the health impact of bronchiectasis in Korea, we need a tool for assessing HRQOL of Korean patients with bronchiectasis.

In the present study, we report on the process of the development of the Korean version of the BHQ (K-BHQ) using a standardized methodology. We also evaluated the validity of the K-BHQ in Korean patients with bronchiectasis.

\section{Materials and Methods}

\section{Study subjects}

Patients with stable bronchiectasis were enrolled from two academic hospitals, the Hanyang University Hospital and the Asan Medical Center, Seoul, Korea. These patients were a part of a patient cohort from an ongoing study, the Korean Multicenter Bronchiectasis Audit and Research Collaboration $(\mathrm{KMBARC})^{10}$. The candidates for enrollment in the KMBARC study were patients in stable condition with bronchiectasis confirmed by chest computed tomography. Baseline clinical information, including the modified Medical Research Council (mMRC) dyspnea scale score, lung function, and exacerba- tion history, was obtained in addition to the K-BHQ and Korean version of the Chronic Obstructive Pulmonary Disease Assessment Test (K-CAT) scores.

\section{Methods}

\section{1) BHQ translation from English into the Korean lan- guage}

We obtained permission for the copyright using the original version of the BHQ from Dr. Surinder Birring of King's College Hospital, UK. A research coordinator performed the 1st translation of the original version of the BHQ into the Korean language with the aid of the Google Translator. The research coordinator obtained feedback regarding the 1st translation version from two Korean patients with bronchiectasis. The two patients understood the meaning of all translations, except for the meaning of the translation for question 4 which read; "In the last 14 days, my chest has felt clear." For question 4, we revised the translation with the addition of a popular word in parentheses. Another coordinator translated the revised version of the Korean language back into English. She was blinded to the original English expression of the BHQ. The questionnaire that was translated back into English was reviewed by the developer, Dr. Surinder Birring. One of the authors in this study compared the questions of the original version with those of the back-translated version in English (Figure 1). The final version of the K-BHQ is given in the Supplementary Material.

\section{2) K-BHQ validation}

The validity was assessed by investigating the relationship between the K-BHQ scores and K-CAT scores. We also inves-

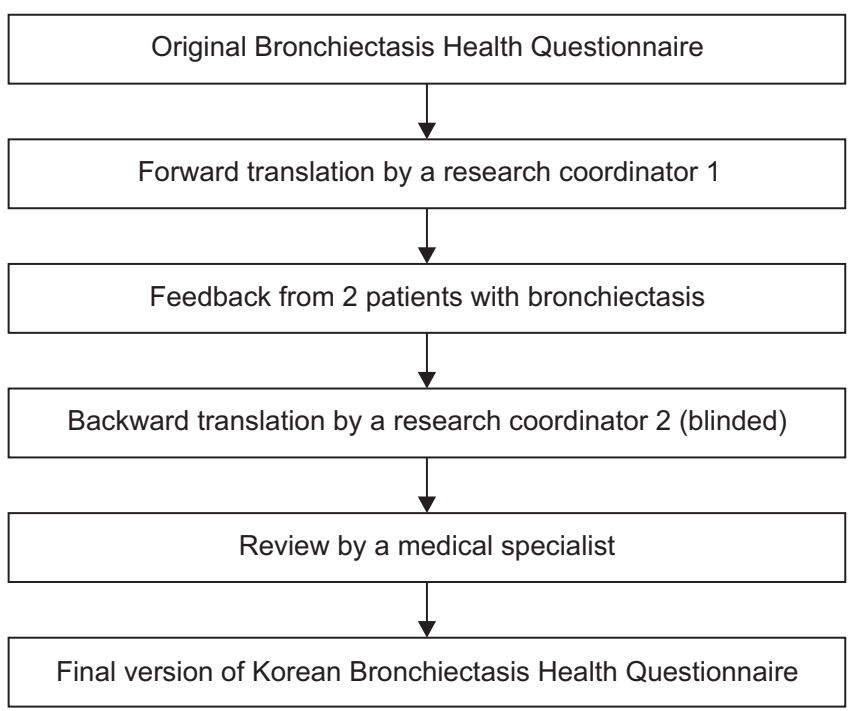

Figure 1. The linguistic validation process of the Korean version of the Bronchiectasis Health Questionnaire. 
Table 1. Characteristics of participants

\begin{tabular}{|c|c|}
\hline Characteristic & Value $(n=126)$ \\
\hline Age, yr & $64.3 \pm 9.7$ \\
\hline Female sex & $67(53.2)$ \\
\hline $\mathrm{BMI}, \mathrm{kg} / \mathrm{m}^{2}$ & $22.7 \pm 3.4$ \\
\hline \multicolumn{2}{|l|}{ Smoking status } \\
\hline Never & $75(59.5)$ \\
\hline Ex & $43(34.1)$ \\
\hline Current & $8(6.3)$ \\
\hline \multicolumn{2}{|l|}{ Previous pulmonary disease history } \\
\hline Tuberculosis & $57(45.2)$ \\
\hline Measles & $30(23.8)$ \\
\hline Nontuberculous mycobacterium & $13(10.3)$ \\
\hline Pertussis & $13(10.3)$ \\
\hline \multicolumn{2}{|c|}{ Prebronchodilator spirometry $(n=106)$} \\
\hline $\mathrm{FEV}_{1} \%$ predicted $(\mathrm{L})$ & $59.6 \pm 18.9(1.62 \pm 0.60)^{*}$ \\
\hline FVC \% predicted (L) & $71.7 \pm 16.6(2.59 \pm 0.80)^{*}$ \\
\hline $\mathrm{FEV}_{1} / \mathrm{FVC} \%$ & $54.4 \pm 25.5$ \\
\hline \multicolumn{2}{|l|}{ mMRC dyspnea scale } \\
\hline 0 & $35(28.8)$ \\
\hline 1 & $65(51.6)$ \\
\hline 2 & $15(11.9)$ \\
\hline 3 & $9(7.1)$ \\
\hline 4 & $2(1.6)$ \\
\hline \multicolumn{2}{|l|}{ Exacerbations in past $12 \mathrm{mo}^{\dagger}$} \\
\hline 0 & $82(65.1)$ \\
\hline 1 & $15(11.9)$ \\
\hline 2 & $15(11.9)$ \\
\hline 3 & $5(4.0)$ \\
\hline 4 & $5(4.0)$ \\
\hline 5 & $4(3.2)$ \\
\hline \multicolumn{2}{|c|}{ Exacerbations requiring hospitalization in last $12 \mathrm{mo}$} \\
\hline 0 & $104(82.5)$ \\
\hline 1 & $14(11.1)$ \\
\hline 2 & $4(3.2)$ \\
\hline 3 & $3(2.4)$ \\
\hline 4 & $1(0.8)$ \\
\hline K-CAT score $(\mathrm{n}=70)$ & $17.6 \pm 9.6$ \\
\hline K-BHQ score $(n=125)$ & $49.0 \pm 9.1$ \\
\hline
\end{tabular}

Values are presented as mean \pm standard deviation (SD) or number (\%) unless otherwise indicated.

*Values are absolute measures of the lung volume given as mean \pm SD. ${ }^{\dagger}$ Any exacerbations that did not require hospital admission. BMI: body mass index; $\mathrm{FEV}_{1}$ : forced expiratory volume in one second; FVC: forced vital capacity; mMRC: modified Medical Research Council; K-CAT: Korean version of Chronic Obstructive Pulmonary Disease Assessment Test; K-BHQ: Korean version of Bronchiectasis Health Questionnaire. tigated the relationship between the K-BHQ scores and other variables of the mMRC dyspnea scale, lung function, and exacerbations.

\section{3) Statistical analysis}

To evaluate the validity of the K-BHQ, correlation analysis was conducted using Pearson's correlation coefficient (r) for the parametric variables and Spearman's correlation coefficient $(\rho)$ for the non-parametric variables.

Statistical analysis was performed using SPSS for Windows version 18.0 (SPSS Inc., Chicago, IL, USA). All data are expressed as means and standard deviations (SD) or frequencies. Statistical significance was accepted for p-values less than 0.05 .

\section{4) Ethics statement}

Approvals were obtained from the institutional review boards (IRBs) of the Asan Medical Center and the Hanyang University Hospital for patient data collection as a component of the cohort study of the KMBARC (IRB No. 2020-0087, 20180691). Written informed consent was obtained from all participants. For this study, we obtained approval from the IRB of the Asan Medical Center.

\section{Results}

\section{Patients}

A total of 126 patients with bronchiectasis were included. The mean $( \pm \mathrm{SD})$ age was $64.3 \pm 9.7$ years, and $53.2 \%$ of the patients were women. Sixty percent of the patients had never smoked, and $38 \%$ had a history of tuberculosis. The mean $( \pm \mathrm{SD})$ forced expiratory volume in 1 second $\left(\mathrm{FEV}_{1}\right)$ and forced vital capacity (FVC) were $60 \pm 19 \%$ and $72 \pm 17 \%$ predicted val-

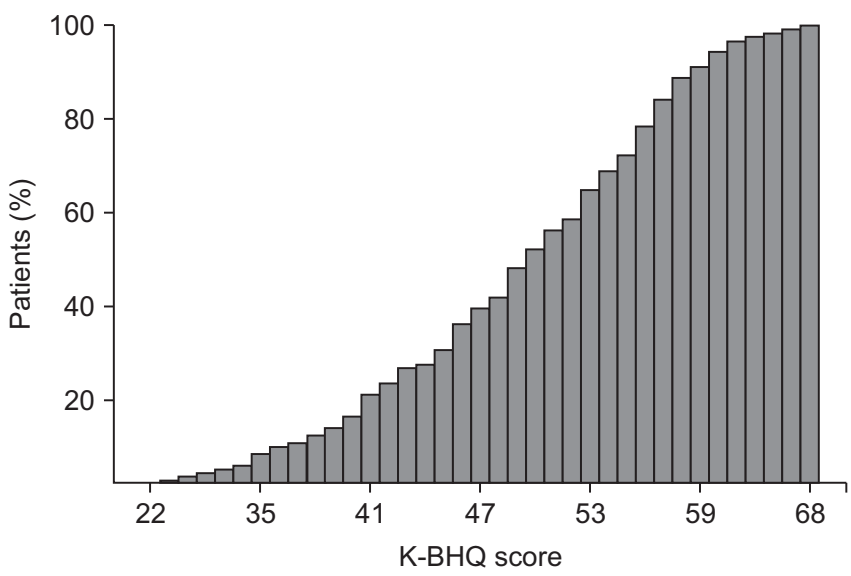

Figure 2. The cumulative frequency distribution of the Korean version of the Bronchiectasis Health Questionnaire (K-BHQ) score in 125 bronchiectasis patients. 
ues, respectively. Most patients did not experience exacerbations or hospitalizations (Table 1).

\section{Correlation between K-BHQ and other clinical measurements}

The mean $( \pm$ SD) K-BHQ total score was 49.0 \pm 9.1 . The scores were evenly distributed from the mean (Figure 2). The correlation between the K-BHQ scores and the K-CAT scores was good $(\mathrm{r}=-0.656, \mathrm{p}<0.001)$ (Figure 3 ). The K-BHQ score correlated moderately with the mMRC dyspnea scale $(\rho=-0.409$, $\mathrm{p}<0.001)$ (Figure 4), $\mathrm{FEV}_{1} \%$ predicted $(\mathrm{r}=0.406, \mathrm{p}<0.001)$, and FVC \% predicted $(\mathrm{r}=0.351, \mathrm{p}<0.001)$ (Figure 5$)$ and weakly correlated with the number of exacerbations requiring hospitalization in the past year $(\rho=-0.303, p=0.001)$ and any exacer-

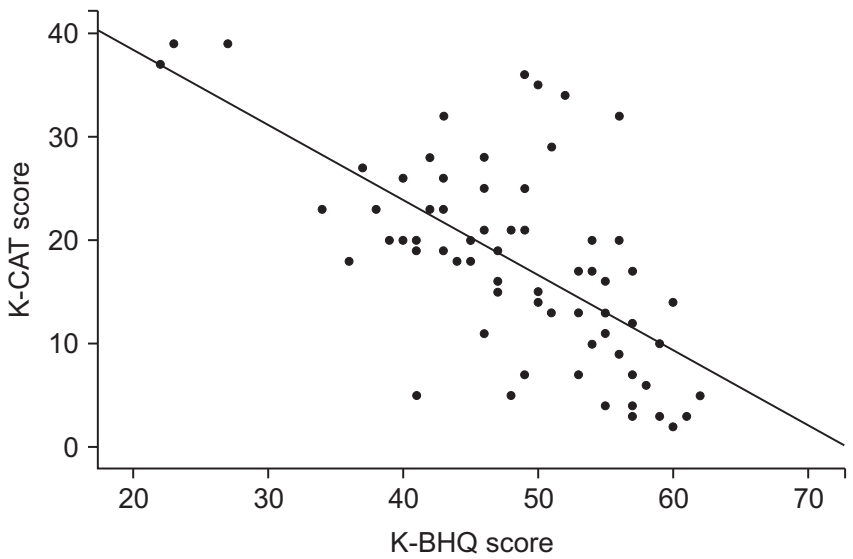

Figure 3. The correlation between the Korean version of the Bronchiectasis Health Questionnaire (K-BHQ) score and the Chronic Obstructive Pulmonary Disease Assessment Test (K-CAT) score in bronchiectasis patients $(\mathrm{r}=-0.656, \mathrm{p}<0.001)$.

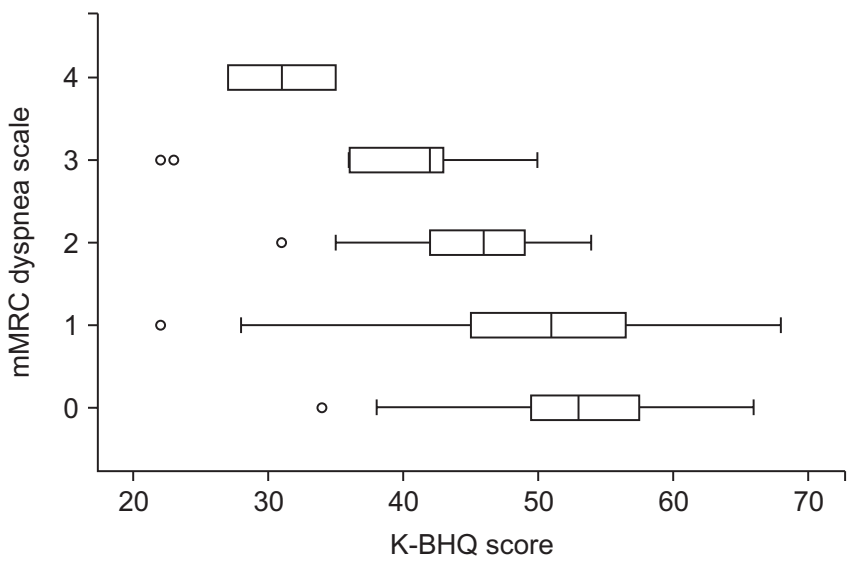

Figure 4. The correlation between the Korean version of the Bronchiectasis Health Questionnaire (K-BHQ) score and the modified Medical Research Council (mMRC) dyspnea scale in bronchiectasis patients $(\rho=-0.409, \mathrm{p}<0.001)$. bations ( $\rho=-0.245, \mathrm{p}=0.006$ ) (Figure 6, Table 2).

\section{Discussion}

The K-BHQ is a translated version of the original BHQ, which is a brief, practical, and well-validated questionnaire for assessing the HRQOL of patients with bronchiectasis in English. This study described the process of translation and demonstrated that the K-BHQ scores correlated strongly with the K-CAT scores and moderately with mMRC dyspnea scale scores, lung function, and exacerbations requiring hospitalization. These results suggest that the K-BHQ is valid for assessing the HRQOL or health status in Korean patients with bronchiectasis.

Although the prevalence of bronchiectasis is high in Korea ${ }^{4}$, there is no tool to properly assess the health status of bron-

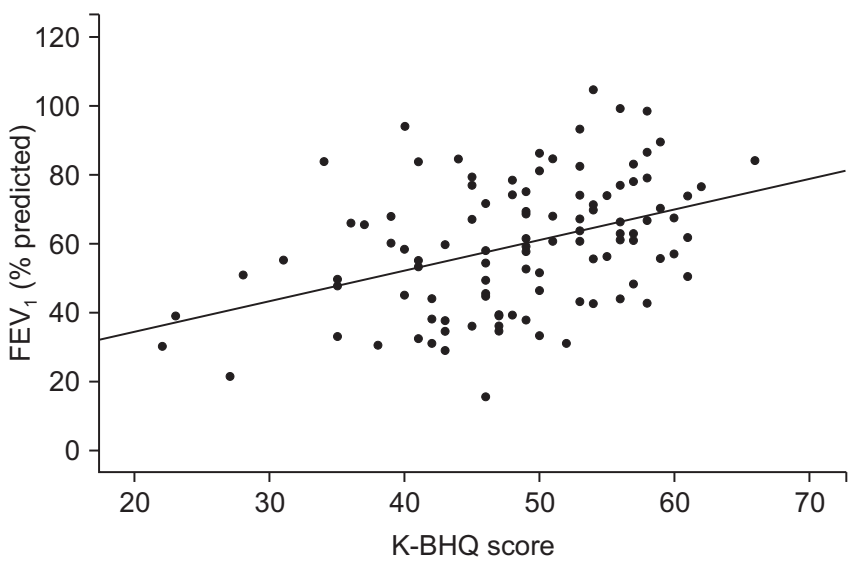

Figure 5. The correlation between the Korean version of the Bronchiectasis Health Questionnaire (K-BHQ) score and forced expiratory volume in one second $\left(\mathrm{FEV}_{1}, \%\right.$ predicted $)(\mathrm{r}=0.406, \mathrm{p}<0.001)$.

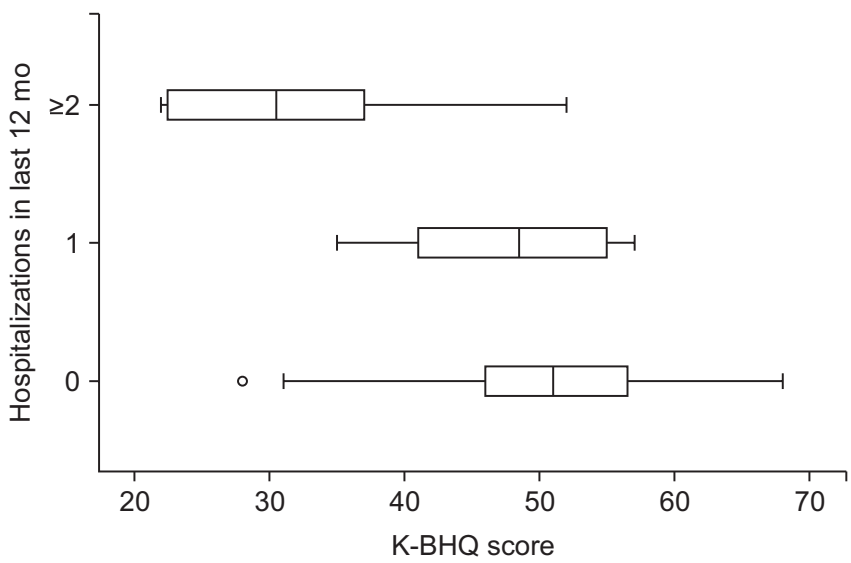

Figure 6. The correlation between the Korean version of the Bronchiectasis Health Questionnaire (K-BHQ) score and exacerbations requiring hospitalization in the previous year $(\rho=-0.303, p=0.001)$. 
Table 2. The correlation between the K-BHQ score and clinical parameters

\begin{tabular}{|lcr|}
\hline & $\begin{array}{c}\text { K-BHQ correlation } \\
\text { coefficient }\end{array}$ & p-value \\
\hline FEV $_{1}, \%$ predicted & $\mathrm{r}=0.406$ & $<0.001$ \\
FVC, \% predicted & $\mathrm{r}=0.351$ & $<0.001$ \\
\hline Exacerbations in last 12 mo & & \\
\hline Any & $\rho=-0.245$ & 0.006 \\
Mild to moderate* & $\rho=-0.116$ & 0.198 \\
Severe $^{\dagger}$ & $\rho=-0.303$ & 0.001 \\
mMRC grade $^{*}$ & $\rho=-0.409$ & $<0.001$ \\
K-CAT score & $\mathrm{r}=-0.656$ & $<0.001$ \\
\hline
\end{tabular}

Correlation coefficients are presented as Pearson's r value (r) and Spearman's $\rho$ value $(\rho)$.

*Any exacerbations that did not require hospitalization. "Exacerbations that required hospitalization.

K-BHQ: the Korean version of the Bronchiectasis Health Questionnaire; $\mathrm{FEV}_{1}$ : forced expiratory volume in one second; $\mathrm{FVC}$ : forced vital capacity; mMRC: modified Medical Research Council; K-CAT: the Korean version of the Chronic Obstructive Pulmonary Disease Assessment Test.

chiectasis patients. A previous study showed that the K-CAT developed for chronic obstructive pulmonary disease (COPD) patients is valid for Korean patients with bronchiectasis ${ }^{11}$. The CAT is a tool for patients with COPD; however, there is no item for hemoptysis, which is a symptom specific to bronchiectasis. In addition, the CAT does not cover the bacterial infection that is common in patients with bronchiectasis. Therefore, we translated the original BHQ developed in British English into Korean and evaluated the validity of the K-BHQ for assessing the HRQOL of patients with bronchiectasis.

The QoL-B is another validated bronchiectasis-specific health status questionnaire ${ }^{5,12}$. The QoL-B has 37 items and 8 domains, while the BHQ consists of 10 items and does not provide an overall quality of life score. The BHQ may, therefore, be more appropriate for clinical and research settings where the implementation of a simple questionnaire is an important practical factor, and when a single score is preferable in order to easily interpret clinical outcomes.

The translation process was based on linguistic validation. Linguistic validation is a series of processes designed to ensure that translations are linguistically accurate, culturally appropriate, and reviewed by experts in the field. The good translation goes beyond simply changing words or word order. Many semantic and cultural specifications must be considered $^{13}$. As much as possible, we attempted to carry out our translation in a manner that reflected the development of the original questionnaire. During the translation process, we were in correspondence with the author of the original BHQ study. The translation proceeded in the following order: (1) in- depth analysis of the original wording and suggestions for suitable translation alternatives, (2) a forward/backward translation step, (3) review of the back translation by the developer, and (4) review of the translated questionnaire by a respiratory clinician fluent in the language ${ }^{9}$.

Compared with the original $\mathrm{BHQ}$, the K-BHQ showed lower convergent validity ( $\mathrm{r}=-0.82$ vs. $\mathrm{r}=-0.66$ ). This is probably because the validity of the original BHQ study was obtained using the SGRQ, while validation in this study was conducted using the K-CAT. The K-BHQ scores are more closely correlated with lung functions and less correlated with exacerbations and hospitalizations than the original BHQ. The reason may be due to differences in patient characteristics. Patients enrolled in the K-BHQ study were older and had lower lung functions, less exacerbations, and more history of tuberculosis than the BHQ study participants.

In addition, a recent correlation study of quality of life in bronchiectasis reported a correlation between CAT and BHQ scores and showed similar results to the correlation between $\mathrm{K}-\mathrm{BHQ}$ and K-CAT in this study ${ }^{14}$.

In summary, the K-BHQ is a simple, practical, and wellvalidated questionnaire for assessing health status in Korean patients with bronchiectasis.

\section{Authors' Contributions}

Conceptualization: Oh YM. Methodology: Oh YM. Formal analysis: Kim HK. Data curation: Lee H, Choi H, Kim SH, Lee JS, Lee SW, Lee JH. Software: Lee H, Choi H, Kim SH, Lee JS, Lee SW, Lee JH. Validation: Kim HK. Investigation: Kim HK. Writing - original draft preparation: Kim HK, Kim SH. Writing - review and editing: Lee H, Choi H, Lee JS, Lee SW, Lee JH, Oh YM. Approval of final manuscript: all authors.

\section{Conflicts of Interest}

No potential conflict of interest relevant to this article was reported.

\section{Acknowledgments}

The authors thank Dr Surrinder S. Birring for allowing us to translate the original BHQ to K-BHQ.

\section{Funding}

This study was supported by a 2018-Grant from the Korean Academy of Tuberculosis and Respiratory Diseases. 


\section{Supplementary Material}

Supplementary material can be found in the journal homepage (http://www.e-trd.org).

\section{References}

1. Polverino E, Goeminne PC, McDonnell MJ, Aliberti S, Marshall SE, Loebinger MR, et al. European Respiratory Society guidelines for the management of adult bronchiectasis. Eur Respir J 2017;50:1700629.

2. Quint JK, Millett ER, Joshi M, Navaratnam V, Thomas SL, Hurst JR, et al. Changes in the incidence, prevalence and mortality of bronchiectasis in the UK from 2004 to 2013: a population-based cohort study. Eur Respir J 2016;47:186-93.

3. Goeminne PC, Hernandez F, Diel R, Filonenko A, Hughes $\mathrm{R}$, Juelich F, et al. The economic burden of bronchiectasis known and unknown: a systematic review. BMC Pulm Med 2019;19:54.

4. Choi H, Yang B, Nam H, Kyoung DS, Sim YS, Park HY, et al. Population-based prevalence of bronchiectasis and associated comorbidities in South Korea. Eur Respir J 2019;54: 1900194.

5. Quittner AL, O'Donnell AE, Salathe MA, Lewis SA, Li X, Montgomery AB, et al. Quality of Life Questionnaire-Bronchiectasis: final psychometric analyses and determination of minimal important difference scores. Thorax 2015;70:12-20.

6. Spinou A, Fragkos KC, Lee KK, Elston C, Siegert RJ, Loebinger MR, et al. The validity of health-related quality of life questionnaires in bronchiectasis: a systematic review and metaanalysis. Thorax 2016;71:683-94.

7. Jones PW, Quirk FH, Baveystock CM, Littlejohns P. A self- complete measure of health status for chronic airflow limitation. The St. George's Respiratory Questionnaire. Am Rev Respir Dis 1992;145:1321-7.

8. Xu Z, Lin R, Che G, Wang M, Ji Y, Li P, et al. Validation of the Mandarin Chinese version of the Leicester Cough Questionnaire in patients undergoing lung resection for patients with lung disease. Zhongguo Fei Ai Za Zhi 2017;20:389-94.

9. Spinou A, Siegert RJ, Guan WJ, Patel AS, Gosker HR, Lee KK, et al. The development and validation of the Bronchiectasis Health Questionnaire. Eur Respir J 2017;49:1601532.

10. Lee H, Choi H, Sim YS, Park S, Kim WJ, Yoo KH, et al. KMBARC registry: protocol for a multicentre observational cohort study on non-cystic fibrosis bronchiectasis in Korea. BMJ Open 2020;10:e034090.

11. Lee BY, Lee S, Lee JS, Song JW, Lee SD, Jang SH, et al. Validity and reliability of CAT and Dyspnea-12 in bronchiectasis and tuberculous destroyed lung. Tuberc Respir Dis 2012;72:46774.

12. Quittner AL, Marciel KK, Salathe MA, O'Donnell AE, Gotfried $\mathrm{MH}$, Ilowite JS, et al. A preliminary quality of life questionnaire-bronchiectasis: a patient-reported outcome measure for bronchiectasis. Chest 2014;146:437-48.

13. Wild D, Grove A, Martin M, Eremenco S, McElroy S, VerjeeLorenz A, et al. Principles of good practice for the translation and cultural adaptation process for Patient-Reported Outcomes (PRO) measures: report of the ISPOR Task Force for Translation and Cultural Adaptation. Value Health 2005;8:94104.

14. De la Rosa Carrillo D, Olveira C, Garcia-Clemente M, GironMoreno RM, Nieto-Royo R, Navarro-Rolon A, et al. COPD assessment test in bronchiectasis: minimum clinically important difference and psychometric validation: a prospective study. Chest 2020;157:824-33. 